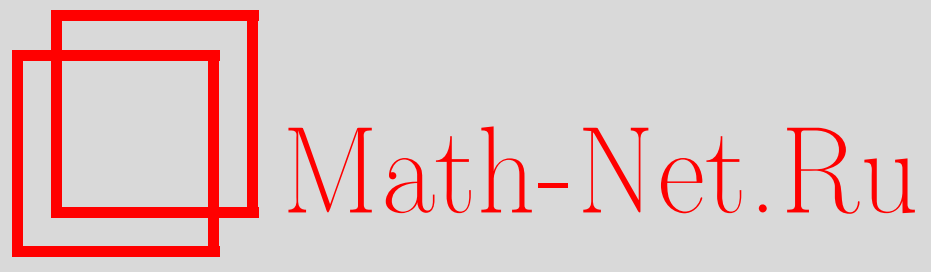

П. А. Крутицкий, О потенциале Стокса для давления, УМH, 2007, том 62, выпуск 2, 177-178

DOI: https://doi.org/10.4213/rm6159

Использование Общероссийского математического портала Math-Net.Ru подразумевает, что вы прочитали и согласны с пользовательским соглашением http://www . mathnet.ru/rus/agreement

Параметры загрузки:

IP: 34.229 .45 .116

26 апреля 2023 г., 11:46:06

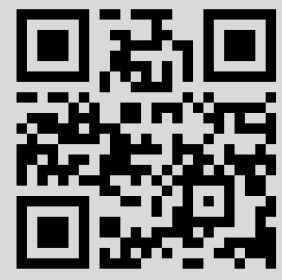




\section{О потенциале Стокса для давления}

\section{П. А. Крутицкий}

Потенциалы для двумерных уравнений Стокса построены и изучены в [1] в случае замкнутых кривых и непрерывных плотностей в потенциалах. В настоящей заметке рассматривается потенциал давления, заданный на разомкнутой кривой. При этом плотности в потенциале принадлежат весовому пространству Гёльдера и могут иметь степенные особенности.

Под разомкнутой кривой понимается простая гладкая незамкнутая дуга конечной длины без самопересечений [2]. В декартовых координатах $x=\left(x_{1}, x_{2}\right) \in \mathbb{R}^{2}$ на плоскости рассмотрим простую гладкую разомкнутую кривую $\Gamma$ класса $\mathbb{C}^{1, \lambda}, \lambda \in(0,1]$, параметризованную длиной дуги $s: \Gamma=\left\{x: x=x(s)=\left(x_{1}(s), x_{2}(s)\right), s \in[a, b]\right\}$. Тем самым, между точками $x \in \Gamma$ и значениями параметра $s$ имеется взаимно однозначное соответствие. Вектор касательной к $\Gamma$ в точке $x(s)$ обозначим $\tau_{s}=(\cos \alpha(s), \sin \alpha(s))$, где $\cos \alpha(s)=x_{1}^{\prime}(s), \sin \alpha(s)=x_{2}^{\prime}(s)$. Отрезок $[a, b]$ будет также обозначаться $\Gamma$.

Пусть вещественнозначная функция $\mu(s)$ класса $\mathbb{C}_{q}^{\omega}[a, b], \omega \in(0,1], q \in[0,1)$, задана на $Г$. Будем говорить, что $\mu(s) \in \mathbb{C}_{q}^{\omega}[a, b]$, если $\mu_{0}(s) \in \mathbb{C}^{0, \omega}[a, b]$, где $\mu_{0}(s)=$ $\mu(s)|s-a|^{q}|s-b|^{q}$ и $\mathbb{C}^{0, \omega}[a, b]-$ класс функций, гёльдеровых с показателем $\omega$.

Рассмотрим $\Gamma$ как разрез на плоскости. Ту сторону разреза $\Gamma$, которая остается слева при возрастании параметра $s$, будем обозначать $\Gamma^{+}$, а противоположную сторону будем обозначать $\Gamma^{-}$. Пусть $\mu_{1}(s), \mu_{2}(s)$ - вещественнозначные интегрируемые функции. Цель настоящей заметки - изучить свойства потенциала давления для уравнений Стокса [1]:

$$
P\left[\mu_{1}, \mu_{2}\right](x)=P_{c}\left[\mu_{1}\right](x)+P_{s}\left[\mu_{2}\right](x),
$$

где

$$
\begin{gathered}
P_{c}\left[\mu_{1}\right](x)=\frac{1}{2 \pi} \int_{\Gamma} \mu_{1}(\sigma) \frac{\cos (\psi(x, y(\sigma)))}{|x-y(\sigma)|} d \sigma, \quad P_{s}\left[\mu_{2}\right](x)=\frac{1}{2 \pi} \int_{\Gamma} \mu_{2}(\sigma) \frac{\sin (\psi(x, y(\sigma)))}{|x-y(\sigma)|} d \sigma, \\
\cos \psi(x, y(\sigma))=\frac{x_{1}-y_{1}(\sigma)}{|x-y(\sigma)|}, \quad \sin \psi(x, y(\sigma))=\frac{x_{2}-y_{2}(\sigma)}{|x-y(\sigma)|} \\
y(\sigma)=\left(y_{1}(\sigma), y_{2}(\sigma)\right) \in \Gamma, \quad|x-y(\sigma)|=\sqrt{\left(x_{1}-y_{1}(\sigma)\right)^{2}+\left(x_{2}-y_{2}(\sigma)\right)^{2}} .
\end{gathered}
$$

Пусть $z=x_{1}+i x_{2}, t=t(\sigma)=\left(y_{1}(\sigma)+i y_{2}(\sigma)\right) \in \Gamma$, тогда $d t=t^{\prime}(\sigma) d \sigma=e^{i \alpha(\sigma)} d \sigma$, $z-t(\sigma)=|x-y(\sigma)| e^{i \psi(x, y(\sigma))}$. Считая, что $\mu(s)$ - вещественная интегрируемая плотность, рассмотрим комплексный потенциал

$$
\begin{aligned}
P_{E}[\mu](x) & =P_{c}[\mu](x)-i P_{s}[\mu](x)=\frac{1}{2 \pi} \int_{\Gamma} \mu(\sigma) \frac{e^{-i \psi(x, y(\sigma))}}{|x-y(\sigma)|} d \sigma \\
& =\frac{1}{2 \pi} \int_{\Gamma} \mu(\sigma) \frac{1}{z-t(\sigma)} d \sigma=\frac{1}{2 \pi} \int_{\Gamma} \mu(\sigma) e^{-i \alpha(\sigma)} \frac{1}{z-t(\sigma)} d t=-\frac{1}{2 \pi} \int_{\Gamma} \widehat{\mu}(t) \frac{1}{t-z} d t,
\end{aligned}
$$

где $\widehat{\mu}(t)=\mu(\sigma) e^{-i \alpha(\sigma)}$. В $P_{E}[\mu](x)$ можно взять либо $\mu \equiv \mu_{1}$, либо $\mu \equiv \mu_{2}$. Тем самым, чтобы изучить функции $P_{c}\left[\mu_{1}\right](x), P_{s}\left[\mu_{2}\right](x)$, достаточно изучить функцию $P_{E}[\mu](x)$ и взять ее действительную или мнимую часть.

Будем говорить, что комплекснозначная функция $\widehat{\mu}(t)$ принадлежит классу $\mathbb{C}_{q}^{\omega}(\Gamma)$ с $\omega \in(0,1], q \in[0,1)$, если $\widehat{\mu}_{0}(t) \in \mathbb{C}^{0, \omega}(\Gamma)$, где $\widehat{\mu}_{0}(t)=\widehat{\mu}(t)|t-t(a)|^{q}|t-t(b)|^{q}$ и $\mathbb{C}^{0, \omega}(\Gamma)$ - класс функций, гёльдеровых с показателем $\omega$. Можно показать, что если $\mu(\sigma) \in \mathbb{C}_{q}^{\omega}[a, b]$, где $\omega \in(0,1], q \in[0,1)$, и если $\widehat{\mu}(t)=\mu(\sigma) e^{-i \alpha(\sigma)}$, то $\widehat{\mu}(t) \in \mathbb{C}_{q}^{\beta}(\Gamma)$, где $\beta=\min \{\omega, \lambda\}$.

Теорема 1. Пусть $Г$ - разомкнутая кривая класса $\mathbb{C}^{1, \lambda}, \lambda \in(0,1], u t=t(\sigma)=$ $\left(y_{1}(\sigma)+i y_{2}(\sigma)\right) \in \Gamma$. Пусть $\mu(\sigma) \in \mathbb{C}_{q}^{\omega}[a, b], \omega \in(0,1], q \in[0,1)$. Тогда

Работа выполнена при поддержке РФФИ (гранты № 05-01-00050, 07-01-00029) и центра Бернулли в Лозанне (Швейцария). 
1) функиия $P_{E}[\mu](x)$ принадлежит $\mathbb{C}^{0}\left(\overline{\mathbb{R}^{2} \backslash \Gamma} \backslash X\right) \cap \mathbb{C}^{2}\left(\mathbb{R}^{2} \backslash \Gamma\right)$, где $X=x(a) \cup x(b)$ множество концов $\Gamma$;

2) предельные значения функиии $P_{E}[\mu](x)$ на $\Gamma^{ \pm}$во внутренних точках даются формулой

$$
\begin{aligned}
\left.P_{E}[\mu](x)\right|_{x(s) \in \Gamma^{ \pm}} & =-i\left( \pm \frac{1}{2} e^{-i \alpha(s)} \mu(s)+\frac{1}{2 \pi i} \int_{\Gamma} \frac{\widehat{\mu}(t)}{t-t_{0}} d t\right) \\
& =-i\left( \pm \frac{1}{2} e^{-i \alpha(s)} \mu(s)-\frac{1}{2 \pi i} \int_{\Gamma} \frac{\mu(s) e^{-i \psi(x(s), y(\sigma))}}{|x(s)-y(\sigma)|} d \sigma\right),
\end{aligned}
$$

где $t_{0}=t_{0}(s)=\left(x_{1}(s)+i x_{2}(s)\right) \in \Gamma$ и интеграл понимается в смысле главного значения;

3) для любой точки $x \notin \Gamma$, расположенной в малой окрестности конца $x(d)(d=a$ или $d=b)$, выполняется неравенство

$$
\left|P_{E}[\mu](x)\right| \leqslant \frac{\text { const }}{|x-x(d)|^{\delta}},
$$

где $\delta=q$, если $q \in(0,1)$, и $\delta$ - произвольное число из интервала $(0,1)$, если $q=0$.

ЗАмечАниЕ. Через $\mathbb{C}^{0}\left(\overline{\mathbb{R}^{2} \backslash \Gamma} \backslash X\right)$ обозначается класс функций, которые непрерывны вне $\Gamma$, непрерывно продолжимы на стороны $\Gamma$ слева и справа во внутренних точках, но предельные значения каждой функции на $Г$ слева и справа могут быть разными.

Теорема 2. Пусть Г-разомкнутая кривая класса $\mathbb{C}^{1, \lambda}, \lambda \in(0,1]$;

$$
\mu(\sigma)=\frac{\eta(\sigma)}{|\sigma-d|^{q}}, \quad q \in(0,1) ; \quad \eta(\sigma) \in \mathbb{C}^{0, \omega}[a, b], \quad \omega \in(0,1] ; \quad d=a \quad \text { или } \quad d=b .
$$

Пусть

$$
\widehat{\nu}(t)=\widehat{\nu}(t(\sigma))=\left(\frac{t(\sigma)-t(d)}{|\sigma-d|}\right)^{q} \eta(\sigma) e^{-i \alpha(\sigma)} .
$$

Тогда $\widehat{\nu}(t) \in \mathbb{C}^{0, \beta}(\Gamma)$, где $\beta=\min \{\omega, \lambda\}$, и для точек $z=x_{1}+i x_{2}$, расположенных вблизи $D=t(d)$ вне $\Gamma$, справедлива асимптотическая формула

$$
P_{E}[\mu](x)=\mp \frac{e^{ \pm q \pi i}}{2 \sin (\pi q)} \frac{\widehat{\nu}(D)}{(z-D)^{q}}+\Theta_{0}(z),
$$

верхний знак берется, если $d=a$, а нижний знак - если $d=b$. Функиия $\Theta_{0}(z)$ непрерывна при $z \rightarrow D$, если $\beta>q$. Если $0<\beta \leqslant q<1$, то для $z$, лежащих вблизи $D=t(d)$ вне $\Gamma$, выполняется неравенство

$$
\left|\Theta_{0}(z)\right| \leqslant \frac{\text { const }}{|z-D|^{q_{1}}},
$$

где $q_{1}$ - произвольное число такое, что $q_{1} \in(q-\beta, q)$. Более того, $\Theta_{0}(z)$ - аналитическая функиия в окрестности точки $D$, разрезанной вдоль Г. Через $(z-D)^{q}$ обозначается ветвъ, аналитическал в окрестности точки $D$, разрезанной вдоль $\Gamma$, u принимающая значение $(t-D)^{q}$ на $\Gamma^{+}$. Кроме того, $\widehat{\nu}(D)=\lim _{\sigma \rightarrow d} \widehat{\nu}(t(\sigma))$, где $\sigma \in(a, b)$.

Теоремы 1, 2 могут быть доказаны непосредственной проверкой с использованием свойств интегралов типа Коши из [2].

\section{Список литературы}

[1] А. Н. Попов, Краевые задачи математической физики. 7: Сб. работ, Тр. МИАН, 116, Наука, Л., 1971, 162-180. [2] Н. И. Мусхелишвили, Сингулярные интегралъные уравнения, Наука, М., 1968.

П. А. Крутицкий (Р. A. Krutitskii)

Институт прикладной математики им. М. В. Келдыша РАН

E-mail: krutitsk@mail.ru
Представлено М. И. Зеликиным Принято редколлегией 22.01.2007 